\title{
Editorial: critical issues in lymphoma management
}

\author{
David Fuchs D Clemens A. Schmitt
}

Received: 7 February 2019 / Accepted: 7 February 2019

(C) Springer-Verlag GmbH Austria, part of Springer Nature 2019

In this issue of memo, a series of short reviews update critical issues in the diagnosis and management of lymphoma.

Drs. Gerlach and Tzankov [1] provide the pathologists' point of view and remind us, first and foremost, of the importance of adequate tissue collection. When performing invasive procedures such as biopsies at diagnosis or at relapse, it is critical to obtain good quality samples and not subject a patient to unnecessary biopsies because of inadequate sample collection. Fine needle aspiration, in particular, does not provide adequate tissue and should not be performed unless there is no other possibility. The authors also stress the need for good communication between clinicians and pathologists and provide a list of crucial information necessary for diagnosis, such as age, location of lymphoma, previous illnesses and medication. All this can help the pathologist to find the correct diagnosis.

Close collaboration of clinicians and pathologists will become even more important in the near future, as deeper insights into the molecular blueprint of our patients' lymphomas by extended histopathological profiling, a growing gene panel of actionable lesions, and, possibly, knowledge on targets of tomorrow's approved rituximab, cyclophosphamide, doxorubicin, vincristine, prednisone (R-CHOP) extensions will be necessary to individualize therapy.

New mutations have to be added to pathologists' routine repertoire because of their therapeutic consequences.

\footnotetext{
D. Fuchs $(\bowtie) \cdot$ C. A. Schmitt

Universitätsklinik für Hämatologie und Internistische

Onkologie, Kepler Universitätsklinikum, Med

Campus III, Johannes Kepler Universität,

Krankenhausstraße 9, 4020 Linz, Austria

david.fuchs@kepleruniklinikum.at
}

A second review [2] presents a glimpse into this future of the treatment of diffuse large B-cell lymphome (DLBCL) and recent advances in therapy, particularly new immune-based therapies in relapsed and refractory disease. Antibody-drug conjugates, emerging combination therapies with checkpoint inhibitors in clinical trials and, of course, CAR-T-cell therapies are discussed.

Novel therapies also bring novel toxicities, which hematologists need to be able to recognize and treat. Dr. Spanberger's review [3] focuses on toxicities of oral agents that are already in routine use, like ibrutinib, idelalisib and venetoclax. He discusses relevant toxicities and their management and provides a concise review of, among others, atrial fibrillation and bleeding with ibrutinib, tumor lysis syndrome with venetoclax or pneumonitis caused by idelalisib.

Conflict of interest D. Fuchs and C.A. Schmitt declare that they have no competing interests.

Publisher's Note Springer Nature remains neutral with regard to jurisdictional claims in published maps and institutional affiliations.

\section{References}

1. Tzankov A, Gerlach MM. Current lymphoma diagnostic standards: the pathologists' view. Memo. 2019; https://doi. org/10.1007/s12254-019-0472-y.

2. Fuchs D. Diffuse large B-cell lymphoma. Memo. 2019; https://doi.org/10.1007/s12254-018-0457-2.

3. Spanberger T. Toxicities in B-cell non-hodgkin lymphoma-new agents, new pitfalls. Memo. 2019; https:// doi.org/10.1007/s12254-018-0466-1. 